\title{
The Neural Correlates of Empathy: Experience, Automaticity, and Prosocial Behavior
}

\author{
Lian T. Rameson, Sylvia A. Morelli, and Matthew D. Lieberman
}

\begin{abstract}
Empathy is a critical aspect of human emotion that influences the behavior of individuals as well as the functioning of society. Although empathy is fundamentally a subjective experience, no studies have yet examined the neural correlates of the self-reported experience of empathy. Furthermore, although behavioral research has linked empathy to prosocial behavior, no work has yet connected empathy-related neural activity to everyday, realworld helping behavior. Lastly, the widespread assumption that empathy is an automatic experience remains largely untested. It is also unknown whether differences in trait empathy reflect either variability in the automaticity of empathic responses or the capacity to feel empathy. In this study, 32 participants completed a diary study of helping behavior followed by an fMRI session, assessing empathic responses to sad images under three
\end{abstract}

\section{INTRODUCTION}

The ability to experience empathy for the plight of our fellow human beings is one of most fundamental skills in the repertoire of human social behavior. Incorporating the cognitive and emotional talents of humans, empathy requires both the ability to comprehend others' thoughts and feelings as well as resonate affectively with their emotions. Empathy is so integral to navigating our world that empathic dysfunction characterizes severe disorders like autism (Farrow \& Woodruff, 2007).

Although empathy is multifaceted, the majority of neuroimaging studies have focused narrowly on the neural response to observing another individual in physical pain. Although recent work has begun to examine other aspects of empathy, such as vicarious reward, vicarious embarrassment, and empathic accuracy (Krach et al., 2011; Mobbs et al., 2009; Zaki, Weber, Bolger, \& Ochsner, 2009), central features of empathy remain entirely absent from neuroscientific investigations. The present research addresses four unstudied aspects of empathy: (1) the neural correlates of the subjective experience of empathy; (2) whether empathy-related neural activity predicts everyday prosocial behavior; (3) whether empathy occurs automatically; and (4) whether high trait empathy reflects a capacity for greater empathic responding or a greater tendency to spontaneously empathize.

University of California—Los Angeles conditions: watching naturally, under cognitive load, and while empathizing. Across conditions, higher levels of self-reported experienced empathy were associated with greater activity in medial PFC (MPFC). Activity in MPFC was also correlated with daily helping behavior. Self-report of empathic experience and activity in empathy-related areas, notably MPFC, were higher in the empathize condition than in the load condition, suggesting that empathy is not a fully automatic experience. Additionally, high trait empathy participants displayed greater experienced empathy and stronger MPFC responses than low trait empathy individuals under cognitive load, suggesting that empathy is more automatic for individuals high in trait empathy. These results underline the critical role that MPFC plays in the instantiation of empathic experience and consequent behavior.

Furthermore, the current study expands on previous research by using naturalistic stimuli that depict a variety of sad events that occur in everyday life. This new experimental paradigm allowed us to examine participants' general empathic response to everyday sad events, rather than a specific response to one type of sad event for a single target (e.g., social rejection for a stranger). By measuring daily prosocial behavior toward friends and strangers, our study is the first to investigate how neural empathy for sadness predicts helping behavior outside an experimental setting for various targets. In addition, whereas previous studies made participants cognitively busy during a neural empathy task (e.g., Gu \& Han, 2007), instructed participants to observe others (e.g., Singer et al., 2004), or to actively imagine how someone else feels (e.g., Lamm, Batson, \& Decety, 2007), no studies have combined these various instructions within one study to determine how empathy may differ while participants are watching naturally, intentionally empathizing, or under cognitive load.

\section{Empathy for Pain}

Nearly all neuroscientific studies of empathy have demonstrated that several regions involved in the experience of physical pain are also activated by observing another individual in pain (e.g., Morrison, Peelen, \& Downing, 2007; Botvinick et al., 2005; Singer et al., 2004). In particular, dorsal ACC (dACC) and anterior insula (AI) have been 
reliably implicated in these studies, and the degree of activation in these areas has been associated with dispositional empathy (Saarela et al., 2007; Singer et al., 2004). These results have been interpreted to suggest that neural "mirroring" provides the mechanism for empathic affective resonance (Decety \& Jackson, 2004). Although this literature has made clear strides in tackling the complex phenomenon of empathy, it remains unknown whether these neural regions are involved in empathy for sadness. As empathy-provoking everyday events often involve sad, rather than painful, stimuli, it is important to discover whether empathy for these experiences recruits similar or distinct neural regions. Furthermore, the emphasis on regions commonly activated by observation and experience has largely precluded the examination of empathic processes unique to observation.

\section{Empathy and MPFC}

Neuropsychiatric evidence suggests that the medial wall of PFC contributes importantly to empathic experience. Investigations of neurodegenerative disease have demonstrated that atrophy of medial PFC (MPFC; BA 10) and dorsomedial PFC (DMPFC; BA 9) is associated with empathic deficits (Rankin et al., 2006; Rankin, Kramer, Mychack, \& Miller, 2003). Similarly, ventromedial PFC (VMPFC; BA 11) lesions are associated with empathic impairment (ShamayTsoory, Tomer, Berger, \& Aharon-Peretz, 2003). Several fMRI studies are concordant with these findings. Empathic judgments have been associated with increased MPFC, DMPFC, and VMPFC activity (Farrow et al., 2001), and a study of empathic accuracy found accurate interpersonal judgments that were associated with activity in MPFC (Zaki et al., 2009). Listening to sad stories has also been associated with activity in DMPFC (Decety \& Chaminade, 2003), whereas VMPFC (Botvinick et al., 2005) and MPFC (Olsson, Nearing, \& Phelps, 2007) have been found in response to observing others in pain.

\section{Experienced Empathy}

Although studies have examined the association between neural activity and trait empathy, none have investigated the neural correlates of the actual experience of empathy. Zaki and colleagues (2009) asked participants to continuously rate how positive or negative they believed targets felt at each moment during emotional video clips, however, participants were not explicitly reporting on how much empathy they were experiencing for the target. Measuring experience constitutes an important method for unambiguously identifying regions associated with empathy. For example, in some studies, it is possible that AI and dACC activity reflects reactions of aversion, disgust, or personal distress, rather than true empathy. Utilizing self-report of the psychological process under study provides a convergent method for identifying the psychological phenomenon underlying neural activity.

\section{Empathy and Helping}

Empathy has been an enduring target of scientific interest due largely to its role in promoting altruistic helping. Although laboratory-based studies have demonstrated a strong relationship between state empathy and prosocial behavior (Batson, 1991), a similar association between trait empathy and prosocial behavior has proven more difficult to establish (Eisenberg \& Miller, 1987). Some researchers have argued that this weak relationship may be because of poor measurement of trait empathy and failure to aggregate across time and situation (Penner, Dovidio, Piliavin, \& Schroeder, 2005; Eisenberg \& Miller, 1987). It has also been suggested that empathy measures that do not rely on self-report (such as fMRI) may better predict prosocial behavior (Marsh, Kozak, \& Ambady, 2007). Furthermore, measuring prosocial behavior using daily experience sampling provides for aggregation of prosocial behaviors in a manner that is less prone to retrospective bias (Bolger, Davis, \& Rafaeli, 2003; Reis \& Gable, 2000).

Although research on the relationship of prosocial behavior and empathy has motivated neuroscientific studies of empathy (Preston \& de Waal, 2002), very little work has connected these two lines of research. One recent study indicated that helping an ingroup member was predicted by activity in AI while observing that individual in pain (Hein, Silani, Preuschoff, Batson, \& Singer, 2010). Another study examining social pain found that activity in AI and MPFC predicted prosocial behavior toward a rejection victim (Masten, Morelli, \& Eisenberger, 2011). A third study found that increased activation in the posterior superior temporal cortex when perceiving the actions and intentions of an agent predicted self-reported altruism (Tankersley, Stowe, \& Huettel, 2007). Some of the previous studies measured how watching a specific stranger during physical or social pain predicted prosocial behavior for the same target but did not measure how neural responses related to more general prosocial tendencies outside the laboratory (Masten et al., 2011; Hein et al., 2010). Tankersley and colleagues (2007) compared neural activity during action perception versus action performance, rather than directly measuring neural activity during empathy. Furthermore, rather than measuring real-world prosocial behavior, participants were asked to predict their own prosocial behavior in hypothetical situations or estimate the frequency of their altruistic behavior. By using daily experience sampling techniques, the current study builds upon the previous studies by examining how neural activity during empathy for sadness predicts prosocial behavior in everyday life toward a variety of targets.

\section{Automaticity of Empathy}

Although the assumption that empathy is a purely automatic process is widespread in the neuroimaging literature, almost no research has subjected empathy paradigms to tests of automaticity, such as the use of cognitive load (Gilbert, Pelham, \& Krull, 1988). The only study to date found that 
dACC and AI activity in a pain paradigm was eliminated when subjects were cognitively busy (Gu \& Han, 2007). Additionally, no studies have manipulated empathy instructions within one study, so it is unknown whether behavioral and neural responses vary across conditions of intentional empathizing, passive viewing, or cognitive load. If empathy is automatic, one would expect comparable activity in all three conditions. If empathy is not purely automatic, the strongest neural responses should be evident in the instructed empathy condition and the weakest under cognitive load.

\section{Empathic Capacity versus Tendency}

It remains unknown whether neural variability is associated with trait empathy because of differences in empathic abilities or habitual tendencies (Berkman \& Lieberman, 2009). On the one hand, high trait empathy individuals might have a greater capacity for empathic experience. If this were true, when instructed to empathize (tapping into maximum capacity), high-empathy individuals should report higher levels of empathy and display divergent neural responses from their low-empathy counterparts. However, if high and low trait empathy individuals differ in spontaneous tendency, one would expect to see differences between groups only when empathy is unprompted.

\section{Overview}

Participants completed a diary study to assess daily helping, followed by an fMRI task utilizing naturalistic stimuli (photos of individuals in sad situations). Stimuli were presented under three conditions: watching naturally, while instructed to empathize, and under cognitive load (memorizing an eightdigit number with no empathy instructions). Participants subsequently rated their empathic concern for targets.

We predicted that participants would display greatest empathy when instructed to empathize, least empathy under load, and intermediate levels while watching naturally. Integrating the fMRI and diary data enabled investigation of whether neural activity predicted variability in daily helping. We predicted that, across analyses, empathy would be associated with activity in MPFC. Specific predictions about dACC and AI were avoided, as these regions have been primarily associated with pain paradigms. For all analyses, both whole-brain and anatomically defined ROI analyses in MPFC, dACC, and AI were conducted. Whole-brain analyses were performed to ensure all brain regions were comprehensively examined, whereas anatomical ROI analyses were conducted in an effort to perform the most stringent, conservative test of research questions (Poldrack \& Mumford, 2009).

\section{METHODS}

\section{Participants}

Informed consent was obtained from 32 healthy, righthanded undergraduates (16 men; mean age $=19.9$ years,
$S D=1.4$ years) who were told that the purpose of the study was to learn how emotion is processed in the brain.

\section{Measures}

Participants were administered the Empathy Quotient (EQ; Baron-Cohen \& Wheelwright, 2004) approximately 1 month before the fMRI session. The EQ measures the affective and cognitive aspects of trait empathy.

\section{Daily Experience Survey}

Participants completed an end-of-day on-line survey for 14 consecutive days. Two forms of daily helping were measured: stranger-acquaintance helping (e.g., picking up dropped objects and holding a door open; $\alpha=.82$ ) and friend helping (e.g., lending money and giving a ride; $\alpha=$.73). See Supplementary Table 1 for complete scales.

\section{fMRI Task}

Participants returned for the fMRI session approximately 21 days $(S D=8.35)$ after completing the diary study. Each block consisted of a contextual sentence describing a sad situation, such as attending a loved one's funeral or being fired from a job, followed by six photos depicting different individuals in that situation. An arrow indicated the target individual if a photo depicted several people. Within each block, half of the targets were men and the other half were women. Images were equated across conditions on arousal, valence, luminance, and complexity, and sentences were equated on length. Images were selected from a larger pool to equate them on a number of features. Blocks were equated across instruction type on arousal, luminance, complexity, and the number of letters in each contextual sentence preceding that block. Subjective ratings of valence and arousal were made by 16 undergraduate pilot judges (eight men). Raters judged the valence of each photo on a scale from 1 (very negative) to 7 (very positive) and arousal on a scale from 1 (very weak) to 7 (very strong). Luminance was measured using Adobe Photoshop CS. Complexity was determined using the size of each image in jpeg (compressed) format. Compressed image file size can be used as a measure of complexity, such that a larger file size may be considered more complex (Calvo \& Lang, 2004). In previous research, compressed image file sizes have been shown to be highly correlated with both subjective measures of complexity (Tuch, Bargas-Avila, Opwis, \& Wilhelm, 2009; Donderi, 2006) and objective visual search performance (Donderi \& McFadden, 2005). See Supplementary Table 2 for means, SDs, and results of one-way ANOVA.

Participants were told that photos depicted real events drawn from news stories, documentaries, and blogs. Nine blocks were divided into three conditions: watch, empathize, and cognitive load. In the watch condition, participants were instructed to respond to the photos naturally, as if they were at home and had come across the images in 
a magazine. For empathize blocks, participants were told to take each target's perspective and imagine how he or she felt about the situation and how it affected his or her life. These instructions have previously been shown to induce empathic concern (Toi \& Batson, 1982). For load blocks, participants were told to keep an eight-digit number in memory while looking at the images.

Sentences and photos were presented for 4 sec each, with 12-sec rest periods separating blocks. The first run consisted exclusively of watch blocks, as this condition was meant to capture unprimed, spontaneous reactions. In the next two runs, participants were cued to trial type by the word "empathize" or "memorize," which appeared for 2 sec after each sentence. In load blocks, an eight-digit number appeared for $3 \mathrm{sec}$ after the "memorize" cue, and a memory test for the number followed each image set. Participants chose between the correct number and a number that was identical except for one digit.

Immediately after scanning, participants rated their empathic reaction to each block. Participants viewed the original task again, but with shorter presentation times (1 sec/image). Participants were told to remember how they felt when they first saw the images and rate how concerned they felt for the targets on a scale from 1 (not at all) to 7 (very much). Participants were told "concerned" meant how compassionate, sympathetic, and moved they felt, as these adjectives have been used to assess empathy in previous research (Toi \& Batson, 1982).

\section{fMRI Acquisition and Data Analysis}

Scanning was performed on a Siemens Trio 3T. Functional images were acquired using an EPI gradient-echo sequence (repetition time $=2000 \mathrm{msec}$, echo time $=30 \mathrm{msec}$, 4- $\mathrm{mm}$ slice thickness $/$ no gap, field of view $=19.2 \mathrm{~cm}$, matrix $=$ $64 \times 64$, flip angle $=90^{\circ}$ ). A T2-weighted structural image was acquired coplanar with the functional images (repetition time $=5000 \mathrm{msec}$, echo time $=34 \mathrm{msec}$, 4-mm slice thickness/no gap, field of view $=19.2 \mathrm{~cm}$, matrix $=128 \times$ 128, flip angle $=90^{\circ}$ ). All images were scalped using the Brain Extraction Tool of FSL (FMRIB Software Library, Oxford University, Oxford, United Kingdom) and realigned within runs using MCFLIRT. Images were then checked for residual motion and noise spikes using a custom automated diagnostic tool (thresholded at 2-mm motion or $2 \%$ global signal change from one image to the next). In SPM8 (Wellcome Department of Imaging Neuroscience, London, United Kingdom), all functional and anatomical images were reoriented to set the origin to the anterior commissure and the horizontal $(y)$ axis parallel to the AC-PC line. Also in SPM8, functional images were realigned within and between runs to correct for residual head motion and COregistered to the matched-bandwidth structural scan using a six-parameter rigid body transformation. The coregistered structural scan was then normalized into Montreal Neurological Institute (MNI) standard stereotactic space using the scalped ICBM152 template, and the resulting parameters were applied to all functional images. Finally, the normalized functional images were resliced into voxels of $3 \mathrm{~mm}^{3}$ and smoothed using an 8-mm FWHM Gaussian kernel.

All single-subject and group analyses were performed in SPM8. First-level effects were estimated using the general linear model and employing a canonical hemodynamic response function convolved with the experimental design. Low-frequency noise was removed using a high-pass filter. Group analyses were conducted using random effects models to enable population inferences (Friston, Holmes, Price, Büchel, \& Worsley, 1999). To keep all conditions as well constrained and equivalent as possible, each condition was modeled using only the $24 \mathrm{sec}$ of image presentation that was invariant across conditions. The remaining trial elements - the instruction prompts, contextual sentences, eight-digit number presentation, and memory test (for memorize blocks) — were modeled separately and were not included in the baseline condition. Whole-brain group-level analyses were performed using an uncorrected $p$ value of $<.005$ with a cluster threshold of 20 . This threshold was chosen to appropriately balance concerns regarding Type I and Type II error (Lieberman, Berkman, \& Wager, 2009; Lieberman \& Cunningham, 2009). This joint threshold produces a false discovery rate that is comparable to the effective false discovery rate in typical behavioral science articles (recomputed here to parallel that of Lieberman \& Cunningham, 2009). ROI analyses were conducted using Marsbar (Brett, Anton, Valabregue, \& Poline, 2002). Anatomical ROIs were constructed in PickAtlas (Maldjian, Laurienti, Kraft, \& Burdette, 2003) using the automated anatomic atlas (Tzourio-Mazoyer et al., 2002). Insula was bounded caudally at $y=0$ (MNI) to include only the anterior region, and cingulate was bounded caudally at $y=0$ and rostrally at $y=36$ to include only the dorsal anterior aspect. The MPFC ROI was manually constructed in FSL view in a voxel-by-voxel fashion, informed by recent meta-analyses and reviews pertaining to MPFC function (both anterior rostral and dorsal aspects; Amodio \& Frith, 2006; Northoff et al., 2006; Steele \& Lawrie, 2004) and using the automated anatomic atlas labeling scheme as implemented in the PickAtlas for comparison and reference. The MPFC ROI was bounded dorsally at $z=26$ to distinguish from DMPFC, ventrally at $z=-10$ to distinguish from VMPFC, laterally at $x= \pm 20$ to include only the medial aspect, and caudally at $y=46$ to exclude anterior cingulate (see Figure 4 for visual depiction of ROIs). For visualization of results, group contrasts were overlaid on a surface representation of the MNI canonical brain using the SPM surfrend toolbox (spmsurfrend.sourceforge.net) and NeuroLens (www.neurolens.org/NeuroLens/Home.html).

\section{RESULTS}

\section{Behavioral Results}

\section{Trait Empathy}

EQ scores ranged from 23 to 70 , with a mean of 46.78 (SD $=$ 11.86). Consistent with previous research, only $9 \%$ of our 
healthy, normal participants scored 30 or below on the EQ, compared with over $80 \%$ of individuals with Asperger's syndrome or high-functioning autism (Baron-Cohen \& Wheelwright, 2004).

\section{Daily Experience Survey}

Participants exhibited a very high level of compliance and completed an average of 13.38 of 14 diary entries $(S D=0.81)$.

\section{fMRI Session}

Accuracy rate was $84 \%$ (SD $=20 \%$ ) for the memory test after each load block. A median split separated high (seven men and nine women) and low (nine men and seven women) trait empathy groups by EQ scores. Highempathy individuals performed better than low-empathy individuals $(M=94 \%$ vs. $74 \%, t(30)=3.27, p<.01)$.

Postscan ratings for three participants were not collected. An ANOVA revealed a main effect of condition on experienced empathy, $F(2,56)=5.51, p<.01$. Participants reported less empathy in the load condition $(M=$ $5.45, S D=1.15)$ than the empathize $(M=5.83, S D=$ $0.78)$ or watch $(M=5.83, S D=0.92)$ conditions. Utilizing trait empathy as a between-subject factor revealed an interaction between condition type and trait empathy, $F(2$, $54)=3.67, p<.05$ (see Figure 1). Further investigation showed that high-empathy individuals reported similar levels of empathy across conditions, such that there was no difference between empathize and watch $(M=6.01 \mathrm{vs}$. $6.00, t(14)=.08, p=n s)$, empathize and load $(M=6.01$ vs. 5.91, $t(14)=.62, p=n s)$, or watch and load $(M=6.00$ vs. 5.91, $t(14)=.54, p=n s)$ conditions. In contrast, lowempathy participants reported less empathy under load $(M=4.95)$ than empathize $(M=5.64, t(14)=3.15, p<$ $.01)$ or watch $(M=5.64, t(14)=2.96, p<.05)$ conditions. Load was the only condition that differed between highand low-empathy groups, $t(27)=2.45, p<.05$. This sug-

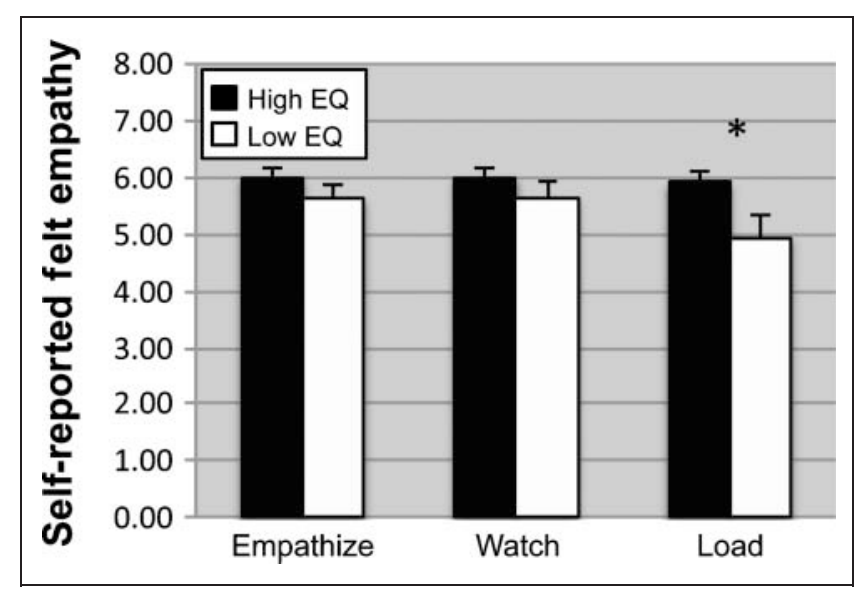

Figure 1. Individuals high in trait empathy experienced more empathy than low-empathy individuals only in the load condition $(p<.05)$. gests that variability in trait empathy reflects differences in empathic tendencies, as high-empathy individuals report greater empathy only in the load condition, where empathizing was unprompted.

\section{fMRI Results}

\section{Experienced Empathy}

To examine the neural correlates of experienced empathy, we created single-subject contrasts by assigning a weight to each block based on the participant's self-reported empathy rating for that block, ignoring condition. These subjectlevel contrasts were then combined into a group contrast to determine regions whose activity tracked self-reported experienced empathy. Interrogation of the three ROIs yielded a significant result in the case of MPFC $(t=2.66$, $p<.01)$, but not dACC $(t=.51, p=n s)$, or AI $(t=.30$, $p=n s)$. In the whole-brain analysis, when self-reports of empathy were stronger, greater activity was observed in MPFC, DMPFC, VMPFC/subgenual ACC (subACC), and ventral striatum (see Figure 2A; Supplementary Table 3).

\section{Daily Helping}

Because help given to friends and strangers differ in nature (Amato, 1990), the two were examined separately. As the load condition produced the most variability in empathic experience, this condition was utilized for these analyses (see Supplementary Data for additional analyses). First, to examine neural regions whose activity is associated with higher daily frequencies of helping friends, each participant's average daily friend helping score was utilized as the regression vector in a group-level regression analysis for the contrast load-fixation. Average daily friend helping was correlated with activity in the MPFC ROI for the contrast load-fixation, $r(30)=.45, p<.01$, as well as in dACC, $r(30)=.39, p<.01$, and AI, $r(30)=.41, p<.01$ This same whole-brain analysis yielded activity in MPFC, DMPFC, precuneus/posterior cingulate (precuneus $_{\text {pcc }}$ ), dACC, insula, nucleus accumbens/caudate, putamen, and lateral temporal cortex (see Figure 2B; Supplementary Table 4). Even controlling for trait empathy (which correlated with daily friend helping, $r(30)=.47, p<.01$ ), activity in the MPFC ROI still predicted daily friend helping, $r(29)=.40, p<.01$, as did activity in dACC, $r(29)=.52, p<.01$, and AI, $r(29)=$ $.57, p<.01$. Conversely, trait empathy was still associated with daily friend helping controlling for MPFC activity, $r(29)=.42, p<.01$, dACC activity, $r(29)=.57, p<.01$, and AI activity, $r(29)=.60, p<.01$. It appears that these two forms of individual difference measurement independently contribute to daily helping.

To examine neural regions whose activity is associated with higher frequencies of helping strangers/acquaintances, each participant's average daily stranger/acquaintance helping score was utilized as the regression vector in a grouplevel regression analysis for the contrast load-fixation. 
Figure 2. (A) Neural regions correlated with self-report of experienced empathy across all conditions included MPFC, DMPFC, subACC, and ventral striatum. (B) Higher levels of daily friend helping were correlated with activity in MPFC, dACC, nucleus accumbens/ caudate, and precuneus for the contrast load-fixation. Daily stranger/acquaintance helping was associated with activity in a region encompassing subACC and MPFC. (C) Neural regions active in the contrast empathize-load included MPFC, DMPFC, VMPFC/subACC, precuneus $_{\mathrm{pcc}}, \mathrm{SMA} /$ midcingulate, and fusiform. (D) Neural regions more active for high trait empathy individuals compared with low-empathy individuals for the contrast load-fixation included MPFC, DMPFC, STS, and VLPFC. All analyses thresholded at $p<.005, k=20$.

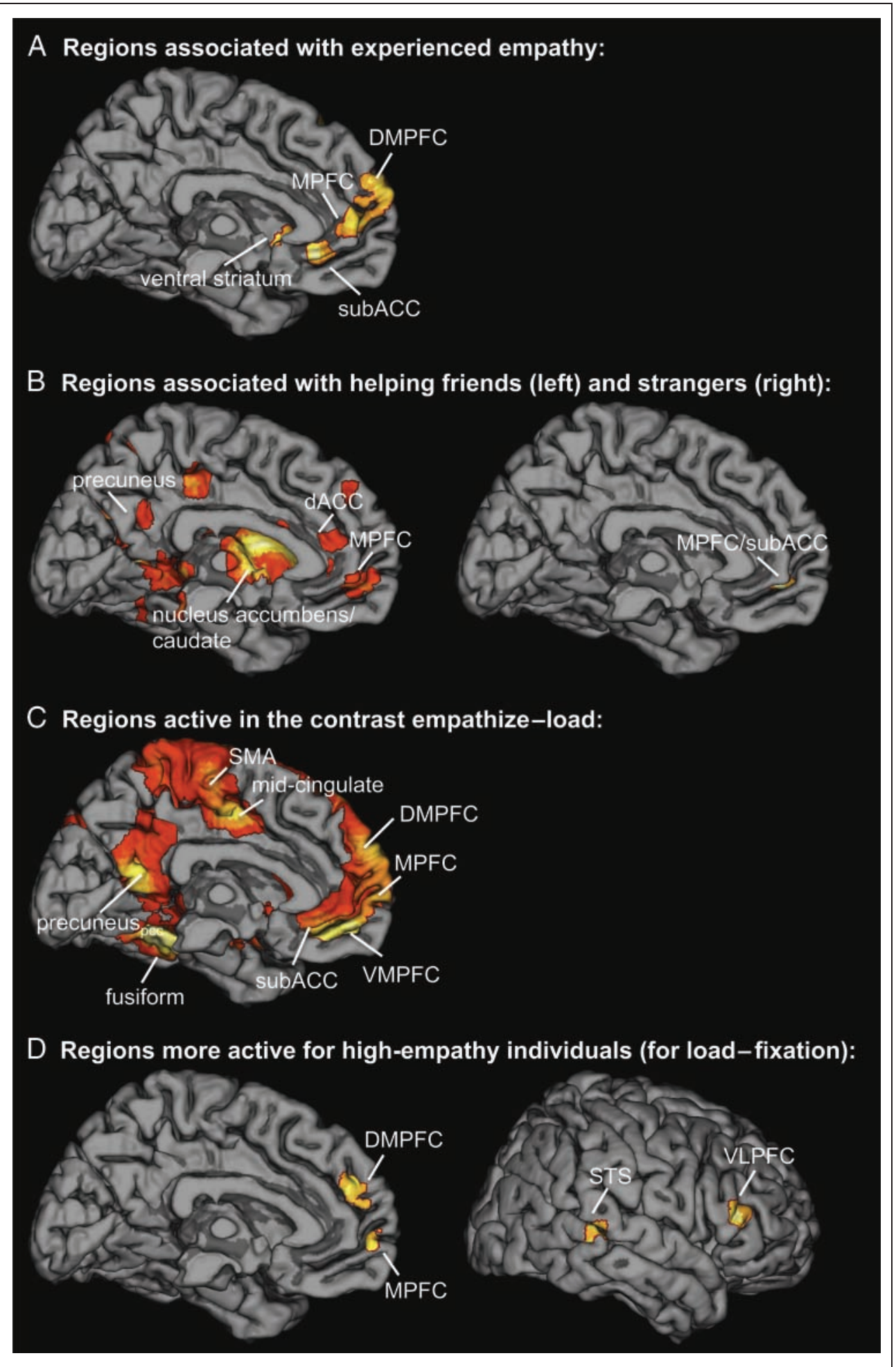

Stranger/acquaintance helping was marginally associated with ROI activity in dACC, $r(30)=.24, p=.09$, but not MPFC, $r(30)=.15, p=n s$, or AI, $r(30)=.09, p=n s$. In the whole-brain analysis, stranger/acquaintance helping was associated with activity in an area encompassing MPFC and subACC $(-18,47,-5)$ and thalamus $(18,-10,-2$; see Figure 2B).

\section{Empatby and Automaticity}

If empathy is a purely automatic process, one would expect no neural differences across the three conditions. However, if empathy is not fully automatic, one would expect to find the greatest empathy-related neural activity while intentionally empathizing, intermediate levels 
while passively viewing (watch condition), and lowest levels when cognitively busy and empathy is unprompted (load condition). ROI analyses for the contrast empathizeload revealed activity in MPFC $(t=4.00, p<.001)$, but not in dACC $(t=-.76, p=n s)$ or AI $(t=-1.01, p=n s)$. The whole-brain analysis yielded activity in MPFC, VMPFC, DMPFC, ventrolateral PFC (VLPFC, pars triangularis, and orbitalis), precuneus $\mathrm{pcc}_{\mathrm{p}}$, pSTS, temporal pole, SMA/midcingulate (see Figure 2C; Supplementary Table 5). For the contrast watch-load, activity was observed in the MPFC ROI $(t=$ $3.03, p<.01)$, but not in dACC $(t=-1.25, p=n s)$ or AI $(t=-1.05, p=n s)$. The whole-brain watch-load analysis produced a set of regions that was nearly identical to the empathize-load contrast, including MPFC (see Supplementary Table 6). The contrast empathize-watch yielded a significant result for the MPFC ROI $(t=1.95, p<.05)$, but not dACC $(t=.47, p=n s)$ or AI $(t=.02, p=n s)$. This whole-brain analysis revealed a set of regions that was very similar to the previous two analyses, including MPFC (see Supplementary Table 7). This suggests that empathy is not a fully automatic process, as intentional empathizing was associated with greater activity in empathy-related regions compared with passive viewing, which in turn was associated with more empathy-related activity than the cognitive load condition.

\section{Trait Empathy}

If individuals high in trait empathy possess greater empathic capacity, their self-report and neural activity should differ from low-empathy participants most when instructed to empathize. However, if high- and low-empathy individuals differ primarily in their spontaneous tendency to empathize, they should diverge most when empathy is uninstructed (watch or load conditions). The self-report data were consistent with a tendency account such that the two groups differed only under load. Two-sample $t$ tests were performed to individually compare the neural activity of high- and low-empathy participants for each condition

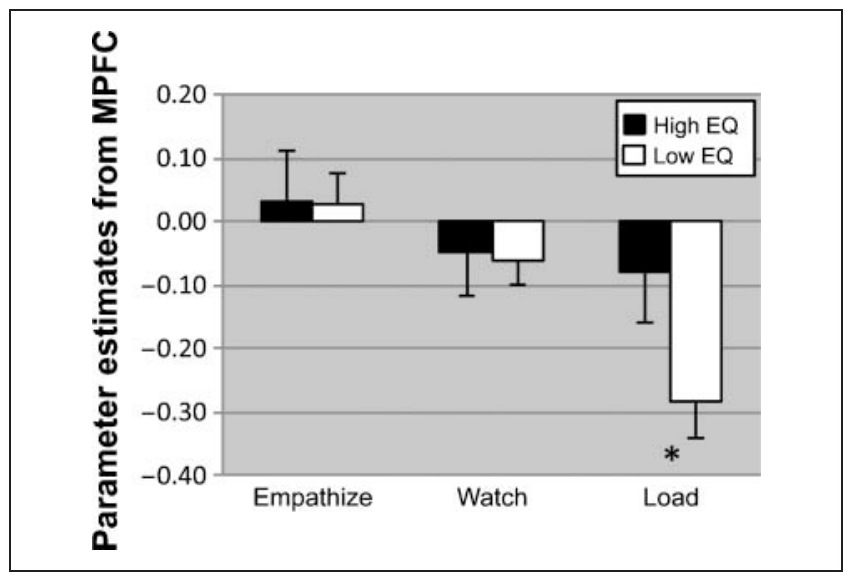

Figure 3. Participants high in trait empathy showed greater activity in the MPFC ROI compared with low-empathy individuals only in the load condition (relative to fixation baseline; $p<.05$ ). (watch, empathize, and load) compared with fixation. Echoing the behavioral results, high-empathy participants displayed significantly more activity than low-empathy participants in the MPFC ROI for the analysis contrasting load-fixation $(t=2.06, p<.05)$. Similar activity was not observed for dACC $(t=-.24, p=n s)$ or AI $(t=-.30, p=n s)$. No activity was observed in any of the ROIs for highempathy compared with low-empathy individuals in the empathize or watch conditions relative to fixation. As with the behavioral self-reported empathy results, an ANOVA (using MPFC activity in each condition compared with fixation as the dependent variable) revealed a significant Condition $\times$ Trait interaction, $F(2,60)=3.25, p<.05$ (see Figure 3). Further investigation using $t$ tests showed that parameter estimates from the MPFC ROI were significantly higher for high-empathy compared with lowempathy individuals only in the load condition $(M=-.08$ vs. $-.28, t(30)=2.05, p<.05)$ and did not significantly differ for the two groups in the empathize ( $M=.03$ vs. .03 , $t(30)=.04, p=n s)$ or watch conditions $(M=-.05$ vs. $-.06, t(30)=.16, p=n s)$. It should be noted that the relative deactivation of MPFC observed compared with fixation (particularly in the load condition) is a commonly observed phenomenon related to default network activity (Raichle et al., 2001), and the relevant comparison here is of relative activity between high- and low-empathy groups.

In the whole-brain analysis, high-empathy participants displayed greater neural activity during load-fixation than those low in empathy in MPFC $(0,59,1)$, DMPFC $(0,47,28)$, VLPFC (pars triangularis; 54,32,13), and posterior STS (63, -40,4; see Figure 2D). In contrast, no regions were more active for high trait empathy participants compared with low trait empathy participants for the contrasts empathizefixation or watch-fixation.

\section{DISCUSSION}

Across ROI analyses, MPFC was reliably associated with the experience of empathy and its behavioral correlates. In contrast, dACC and AI were rarely observed, perhaps an unsurprising result, given that our stimuli did not involve pain (see Figure 4 for a summary of ROI results). In wholebrain analyses, a relatively consistent set of regions were associated with empathic processes, including MPFC, DMPFC, VMPFC/subACC, STS, precuneus ${ }_{\mathrm{pcc}}$, and lateral parietal regions.

\section{Experienced Empathy}

The current results represent the first time that the neural correlates of experienced empathy have been directly identified. We believe that this is an important departure from previous work, in that trait measures have previously been used as a proxy for experienced empathy. To the extent that we want to identify the neural regions associated with a psychological process, it is most accurate to directly measure that process. Higher levels of experienced 


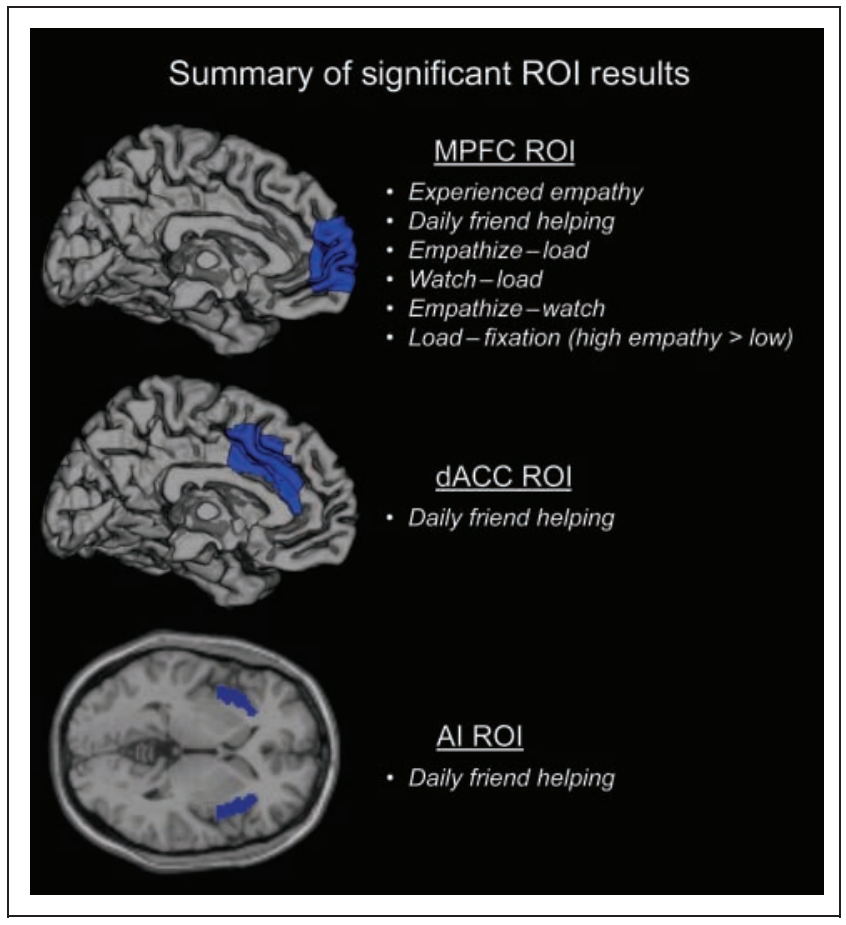

Figure 4. Nearly all empathy-related analyses were associated with significant activity in the MPFC ROI, whereas activity in the dACC and AI ROIs were only associated with daily friend helping.

empathy were associated with activity in MPFC, DMPFC, subACC/VMPFC, and ventral striatum.

\section{Helping}

Both forms of helping were associated with activity in MPFC for the contrast comparing load to fixation. Activity in MPFC predicted friend helping even controlling for trait empathy, suggesting this relationship is not merely the byproduct of shared variance. This is the first study to demonstrate an association between empathy-related neural activity and everyday, real-world helping behavior. Daily friend helping was also associated with activity in dACC and AI for this contrast, which might suggest that a more embodied empathic reaction could motivate helping within the context of friendship. One potential caveat to these results is the finding that the same analyses performed for the watch and empathize conditions compared with fixation did not fully replicate these results (see Supplementary Data). We believe that this divergence is probably because of the relatively restricted range of variability in these two conditions compared with the load condition.

\section{Empathy and Automaticity}

If empathy were fully automatic, one would expect to find no differences across conditions, as automatic processes are typically unaffected by cognitive inducements or interference. We instead found that cognitive load reduced empathic experience and empathy-related neural responses when our participants were considered as a group, suggesting that empathy is not automatic for all people. Furthermore, stronger neural responses were observed when participants were instructed to empathize, which suggests topdown effortful cognition may amplify empathic responses.

\section{Capacity versus Tendency}

The present study parsed variability in trait empathy as a function of capacity or habitual tendency. Our results do not support the capacity account, as no differences were observed between the two groups in the empathize condition. Instead, we observed a Trait $\times$ Situation interaction, such that high-empathy individuals displayed greater experienced empathy and stronger neural responses than low individuals only when under load, suggesting that high-empathy individuals differ in their spontaneous tendency to experience empathy. As high-empathy individuals exhibited superior performance on the memory test, their greater empathic experience cannot be because of less engagement in the load task. A tendency account also predicts differences between the two groups in the watch condition, which was not observed. We speculate that the failure of the two groups to differentiate during the watch condition was the result of the highly negative nature of the stimuli, which seem to have produced large, relatively homogenous empathic responses in the watch condition. Additionally, the population under study was normal, healthy individuals without a history of major psychiatric illness, which may have limited variability in trait empathy. We hypothesize that utilizing either less intense stimuli or a sample with greater variability in trait empathy would result in divergences in the watch condition as well.

These results suggest a novel account of how trait empathy influences empathy in everyday life. When attentional resources are abundant, trait empathy may not impact empathic processes for normal individuals. However, when attention is constrained (as is common during hectic everyday life), the automaticity of empathy for high trait empathy individuals may sustain empathic experience. These findings also add a layer of nuance to the automaticity results: although empathy may not be universally automatic, it appears to be more automatic for individuals high in trait empathy.

\section{Empathy and MPFC}

Across analyses, the consistency with which MPFC was observed suggests that this region plays an important role in empathic processes. This finding is consistent with neuropsychiatric evidence and dovetails nicely with an extensive literature documenting the role of MPFC in social cognition. MPFC has been associated with thinking about one's past (Gilboa, 2004) and possible future experiences (Mason, Bar, \& Macrae, 2009), two processes pivotal to constructing a window into another's emotional world. 
In fact, many participants reported recalling similar events from their own lives to use as a template for understanding, as well as imagining how they might feel if they were in that situation. MPFC is consistently activated in mentalizing or in theory of mind tasks where participants infer the mental states of others (Frith \& Frith, 2006). MPFC has also been associated with thinking about those we feel similar or close to (Krienen, Tu, \& Buckner, 2010; Mitchell, Macrae, \& Banaji, 2006) or just those that we think of as distinctly human (Harris \& Fiske, 2006). Manipulations of these attributes have frequently been employed in behavioral research to induce empathy (Batson, 1991). MPFC was also associated with higher levels of daily helping, which suggests that this region contributes to empathy-related prosocial behavior in everyday life. It is clear that MPFC is central to many social cognitive processes and may be activated across analyses because participants draw on these related processes (e.g., mentalizing) while empathizing. Thus, we highlight that empathic processes consistently activate MPFC and may draw on the same neural regions generally involved in inferring the mental states of others.

\section{Empathy and Other Regions}

Several other regions were also consistently associated with empathic processes. Activity in subACC, which has been associated with sadness (Mayberg et al., 1999), may reflect the experience of congruent sad emotion. Empathic processes were also associated with activity in precuneus ${ }_{\text {pcc }}$, which has been shown in some studies to be involved in mentalizing or the ability to infer the thoughts and feelings of other individuals (for a review, see Lieberman, 2010). This kind of mental process is likely essential to accomplishing the task of affective perspective taking, in which participants were asked to engage in. Activity in VLPFC may suggest the regulation of emotion (Berkman \& Lieberman, 2009), which is thought to be important for avoiding overarousal and personal distress (Eisenberg, 2000). STS activity may reflect the recognition of emotional expression (Narumoto, Okada, Sadato, Fukui, \& Yonekura, 2001). Activity in parietal mirror regions coheres with the notion that neural mirroring facilitates bottom-up emotion matching (Iacoboni, 2009). Ventral striatum, a region linked to reward reinforcement (Cardinal, Parkinson, Hall, \& Everitt, 2002), was associated with self-reported empathy and friend helping. This raises the interesting possibility that individuals may find empathy to be a rewarding experience. Perhaps the sense of emotional connection gained from empathizing is rewarding or possibly the vicarious experience of another's emotion is reinforcing, akin to the pleasure of watching a sad movie.

\section{Limitations}

One potential limitation of the current study design was the presentation of the watch condition in the first run, preceding the presentation of the other two conditions.
Because the watch condition was meant to capture participants' completely spontaneous reactions to the emotional stimuli, we felt presenting it first was imperative to avoiding unwanted interference from the other instruction types. As is often the case, however, emphasizing ecological validity comes at the cost of experimental control, and this design produces an order confound. We attempted to minimize the effect of this cofound through careful prerating to insure that all three conditions were otherwise as equivalent as possible. Follow-up studies in which all three conditions are intermixed will be useful in determining what, if any, effect the presentation order exerted upon the watch condition.

A second possible limitation of the current study is the relatively small number of trials per condition (three blocks featuring 24 sec of image presentation each). The number of blocks was intentionally kept relatively small to avoid habituation and fatigue effects, particularly in light of the emotive content of the stimuli and demanding nature of the task. Although we believe the stimulus presentation was adequate for observing strong effects, it remains possible that activity in certain regions, such as dACC or AI, were not observed because the current study lacked sufficient power to detect such effects.

\section{Implications}

The current findings hold some intriguing implications for clinical research and treatment. It would be interesting to examine whether individuals who suffer from psychiatric disorders, such as autism or psychopathy, differ from healthy controls (and each other) on this task, which allows for the separation of empathic capacity and tendency. Psychopaths might look relatively similar to controls in the empathize condition (which assesses capacity) and diverge sharply in the other two conditions (which tap tendencies). In contrast, individuals with autism might show a pattern that differs from controls across all conditions. This conceptualization of trait empathy suggests that if empathic dysfunction in a particular mental disorder is characterized by habitual tendency, treatment programs aimed at increasing the automaticity of empathic responses might prove effective.

Social psychology has traditionally been concerned about the practical consequences of empathy, but this study constitutes one of the first attempts within the neuroscientific literature on empathy to create this important brain-behavior link. Additional targets for this line of research include whether empathy-related neural activity is positively associated with daily experiences of empathy or negatively associated with behaviors like aggression. Investigating the neural correlates of empathy for positive stimuli has begun (Mobbs et al., 2009) but remains an important target for future research.

As we expand our mapping of brain regions involved in empathic processes, it will be essential to continue to investigate the neural correlates of different forms of empathic 
experience, as well as how these processes unfold during the course of daily experience. Such investigations inform our understanding of the nature and operation of the multifaceted construct of empathy as well as suggest novel approaches to treating disorders characterized by debilitating empathic dysfunction.

\section{Acknowledgments}

We are grateful to Naomi Eisenberger, Shelley Taylor, Annette Stanton, and Marco Iacoboni for their helpful feedback on earlier versions of this manuscript. Austin Grinberg and Kenny Casebere were instrumental in the data collection process. We thank Will Moore and the UO Developmental Social Neuroscience Laboratory for providing the MPFC ROI. We also appreciate the support provided by the University of California-Los Angeles Brainmapping Center. This work was supported by a National Science Foundation Fellowship awarded to L. R.

Reprint requests should be sent to Matthew D. Lieberman, Franz Hall, University of California-Los Angeles, Los Angeles, CA 90095-1563, or via e-mail: mdlieber99@gmail.com.

\section{REFERENCES}

Amato, P. R. (1990). Personality and social network involvement as predictors of helping behavior in everyday life. Social Psychology Quarterly, 53, 31-43.

Amodio, D. M., \& Frith, C. D. (2006). Meeting of minds: The medial frontal cortex and social cognition. Nature Reviews Neuroscience, 7, 268-277.

Baron-Cohen, S., \& Wheelwright, S. (2004). The Empathy Quotient: An investigation of adults with Asperger syndrome or high functioning autism, and normal sex differences. Journal of Autism and Developmental Disorders, 34, 163-175.

Batson, C. D. (1991). The altruism question: Toward a social psychological answer. Hillsdale, NJ: Erlbaum.

Berkman, E. T., \& Lieberman, M. D. (2009). Using neuroscience to broaden emotion regulation: Theoretical and methodological considerations. Social and Personality Psychology Compass, 3, 475-493.

Bolger, N., Davis, A., \& Rafaeli, E. (2003). Diary methods: Capturing life as it is lived. Annual Review of Psychology, 54, 579-616.

Botvinick, M., Jha, A. P., Bylsma, L. M., Fabian, S. A., Solomon, P. E., \& Prkachin, K. M. (2005). Viewing facial expressions of pain engages cortical areas involved in the direct experience of pain. Neuroimage, 25, 312-319.

Brett, M., Anton, J. L., Valabregue, R., \& Poline, J. B. (2002). Region of interest analysis using an SPM toolbox.

Paper presented at the 8th International Conference on Functional Mapping of the Human Brain, Sendai, Japan.

Calvo, M. G., \& Lang, P. J. (2004). Gaze patterns when looking at emotional pictures: Motivationally biased attention. Motivation and Emotion, 28, 221-243.

Cardinal, R., Parkinson, J., Hall, J., \& Everitt, B. (2002). Emotion and motivation: The role of the amygdala, ventral striatum, and prefrontal cortex. Neuroscience \& Biobehavioral Reviews, 26, 321-352.

Decety, J., \& Chaminade, T. (2003). Neural correlates of feeling sympathy. Neuropsychologia, 41, 127-138.

Decety, J., \& Jackson, P. L. (2004). The functional architecture of human empathy. Behavioral and Cognitive Neuroscience Reviews, 3, 71-100.
Donderi, D. C. (2006). Visual complexity: A review. Psychological Bulletin, 132, 73-97.

Donderi, D. C., \& McFadden, S. (2005). Compressed file length predicts search time and errors on visual displays. Displays, 26, 71-78.

Eisenberg, N. (2000). Emotion, regulation, and moral development. Annual Review of Psychology, 51, 665-697.

Eisenberg, N., \& Miller, P. A. (1987). The relation of empathy to prosocial and related behaviors. Psychological Bulletin, 101, 91-119.

Farrow, T. F., \& Woodruff, P. W. R. (2007). Empathy in mental illness. New York: Cambridge University Press.

Farrow, T. F., Zheng, Y., Wilkinson, I. D., Spence, S. A., Deakin, J. F., Tarrier, N., et al. (2001). Investigating the functional anatomy of empathy and forgiveness. NeuroReport, 12, $2433-2438$

Friston, K. J., Holmes, A. P., Price, C. J., Büchel, C., \& Worsley, K. J. (1999). Multisubject fMRI studies and conjunction analyses. Neuroimage, 10, 385-396.

Frith, C. D., \& Frith, U. (2006). The neural basis of mentalizing. Neuron, 50, 531-534.

Gilbert, D., Pelham, B., \& Krull, D. (1988). On cognitive busyness: When person perceivers meet persons perceived. Journal of Personality and Social Psychology, 54, 733-740.

Gilboa, A. (2004). Autobiographical and episodic memoryOne and the same? Evidence from prefrontal activation in neuroimaging studies. Neuropsychologia, 42, 1336-1349.

Gu, X., \& Han, S. (2007). Attention and reality constraints on the neural processes of empathy for pain. Neuroimage, 36, 256-267.

Harris, L. T., \& Fiske, S. T. (2006). Dehumanizing the lowest of the low: Neuroimaging responses to extreme out-groups. Psychological Sciences, 17, 847-853.

Hein, G., Silani, G., Preuschoff, K., Batson, C. D., \& Singer, T. (2010). Neural responses to ingroup and outgroup members' suffering predict individual differences in costly helping. Neuron, 68, 149-160.

Iacoboni, M. (2009). Imitation, empathy, and mirror neurons. Annual Review of Psychology, 60, 653-670.

Krach, S., Cohrs, J. C., de Echeverría Loebell, N. C., Kircher, T., Sommer, J., Jansen, A., et al. (2011). Your flaws are my pain: Linking empathy to vicarious embarrassment. PLOS ONE, 6, e18675.

Krienen, F. M., Tu, P. C., \& Buckner, R. L. (2010). Clan mentality: Evidence that the medial prefrontal cortex responds to close others. Journal of Neuroscience, 30, 13906-13915.

Lamm, C., Batson, C. D., \& Decety, J. (2007). The neural substrate of human empathy: Effects of perspective-taking and cognitive appraisal. Journal of Cognitive Neuroscience, 19, 42-58.

Lieberman, M. D. (2010). Social cognitive neuroscience. In S. T. Fiske, D. T. Gilbert, \& G. Lindzey (Eds.), Handbook of social psychology (5th ed., pp. 143-193). New York: McGraw-Hill.

Lieberman, M. D., Berkman, E. T., \& Wager, T. D. (2009). Correlations in social neuroscience aren't voodoo: Commentary on Vul et al. (2009). Perspectives on Psychological Science, 4, 299-307.

Lieberman, M. D., \& Cunningham, W. A. (2009). Type I and type II error concerns in fMRI research: Re-balancing the scale. Social Cognitive and Affective Neuroscience, 4, 423-428.

Maldjian, J., Laurienti, P., Kraft, R., \& Burdette, J. (2003). An automated method for neuroanatomic and cytoarchitectonic atlas-based interrogation of fMRI data sets. Neuroimage, 19, 1233-1239.

Marsh, A. A., Kozak, M. N., \& Ambady, N. (2007). Accurate identification of fear facial expressions predicts prosocial behavior. Emotion, 7, 239-251. 
Mason, M. F., Bar, M., \& Macrae, C. N. (2009). Exploring the past and impending future in the here and now: Mind-wandering in the default state. Cognitive Sciences, 3, 143-162.

Masten, C. L., Morelli, S. A., \& Eisenberger, N. I. (2011). An fMRI investigation of empathy for "social pain" and subsequent prosocial behavior. Neuroimage, 55, 381-388.

Mayberg, H. S., Liotti, M., Brannan, S. K., McGinnis, S., Mahurin, R. K., Jerabek, P. A., et al. (1999). Reciprocal limbic-cortical function and negative mood: Converging PET findings in depression and normal sadness. American Journal of Psychiatry, 156, 675-682.

Mitchell, J. P., Macrae, C. M., \& Banaji, M. R. (2006). Dissociable medial prefrontal contributions to judgments of similar and dissimilar others. Neuron, 50, 655-663.

Mobbs, D., Yu, R., Meyer, M., Passamonti, L., Seymour, B., Calder, A. J., et al. (2009). A key role for similarity in vicarious reward. Science, 324, 900.

Morrison, I., Peelen, M. V., \& Downing, P. E. (2007). The sight of others' pain modulates motor processing in human cingulate cortex. Cerebral Cortex, 17, 2214-2222.

Narumoto, J., Okada, T., Sadato, N., Fukui, K., \& Yonekura, Y. (2001). Attention to emotion modulates fMRI activity in human right superior temporal sulcus. Cognitive Brain Research, 12, 225-231.

Northoff, G., Heinzel, A., de Greck, M., Bermpohl, F., Dobrowolny, H., \& Panksepp, J. (2006). Self-referential processing in our brain-A meta-analysis of imaging studies on the self. Neuroimage, 31, 440-457.

Olsson, A., Nearing, K. I., \& Phelps, E. A. (2007). Learning fears by observing others: The neural systems of social fear transmission. Social Cognitive and Affective Neuroscience, 2, 3-11.

Penner, L. A., Dovidio, J. F., Piliavin, J. A., \& Schroeder, D. A. (2005). Prosocial behavior: Multilevel perspectives. Annual Review of Psychology, 56, 365-392.

Poldrack, R. A., \& Mumford, J. A. (2009). Independence in ROI analysis: Where is the voodoo? Social Cognitive and Affective Neuroscience, 4, 208-213.

Preston, S. D., \& de Waal, F. B. (2002). Empathy: Its ultimate and proximate bases. Behavioral and Brain Sciences, 25, 1-20; discussion 20-71.

Raichle, M. E., MacLeod, A. M., Snyder, A. Z., Powers, W. J., Gusnard, D. A., \& Shulman, G. L. (2001). A default mode of brain function. Proceedings of the National Academy of Sciences, U.S.A., 98, 676-682.

Rankin, K. P., Gorno-Tempini, M. L., Allison, S. C., Stanley, C. M., Glenn, S., Weiner, M. W., et al. (2006). Structural anatomy of empathy in neurodegenerative disease. Brain, 129, 2945-2956.

Rankin, K. P., Kramer, J. H., Mychack, P., \& Miller, B. L. (2003). Double dissociation of social functioning in frontotemporal dementia. Neurology, 60, 266-271.

Reis, H. T., \& Gable, S. L. (2000). Event-sampling and other methods for studying everyday experience. In $\mathrm{H}$. T. Reis \& C. M. Judd (Eds.), Handbook of research methods in social and personality psychology (pp. 190-222). New York: Cambridge University Press.

Saarela, M. V., Hlushchuk, Y., Williams, A. C., Schurmann, M., Kalso, E., \& Hari, R. (2007). The compassionate brain: Humans detect intensity of pain from another's face. Cerebral Cortex, 17, 230-237.

Shamay-Tsoory, S. G., Tomer, R., Berger, B. D., \& Aharon-Peretz, J. (2003). Characterization of empathy deficits following prefrontal brain damage: The role of the right ventromedial prefrontal cortex. Journal of Cognitive Neuroscience, 15, 324-337.

Singer, T., Seymour, B., O’Doherty, J., Kaube, H., Dolan, R. J., \& Frith, C. D. (2004). Empathy for pain involves the affective but not sensory components of pain. Science, 303, 1157-1162.

Steele, J. D., \& Lawrie, S. M. (2004). Segregation of cognitive and emotional function in the prefrontal cortex: A stereotactic meta-analysis. Neuroimage, 21, 868-875.

Tankersley, D., Stowe, J. C., \& Huettel, S. A. (2007). Altruism is associated with an increased neural response to agency. Nature Neuroscience, 10, 150-151.

Toi, M., \& Batson, C. (1982). More evidence that empathy is a source of altruistic motivation. Journal of Personality and Social Psychology, 43, 281-292.

Tuch, A. N., Bargas-Avila, J. A., Opwis, K., \& Wilhelm, F. H. (2009). Visual complexity of websites: Effects on users' experience, physiology, performance, and memory. International Journal of Human-Computer Studies, 67, 703-715.

Tzourio-Mazoyer, N., Landeau, B., Papathanassiou, D., Crivello, F., Etard, O., Delcroix, N., et al. (2002). Automated anatomical labeling of activations in SPM using a macroscopic anatomical parcellation of the MNI MRI single-subject brain. Neuroimage, 15, 273-289.

Zaki, J., Weber, J., Bolger, N., \& Ochsner, K. N. (2009). The neural bases of empathic accuracy. Proceedings of the National Academy of Sciences, U.S.A., 106, 11382-11387. 